\title{
Bar Galaxies and their Environments
}

\author{
Sidney van den Bergh \\ Dominion Astrophysical Observatory, Herzberg Institute of Astrophysics, National \\ Research Council of Canada, 5071 West Saanich Road, Victoria, BC, V9E 2E7, Canada \\ sidney . vandenbergh@nrc.ca
}

Received __; accepted _ _ 


\begin{abstract}
The prints of the Palomar Sky Survey, luminosity classification and radial velocities were used to assign all northern Shapley-Ames galaxies to either (1) field, (2) group, or (3) cluster environments. This information for 930 galaxies shows no evidence for a dependence of bar frequency on galaxy environment. This suggests that the formation of a bar in a disk galaxy is mainly determined by the properties of the parent galaxy, rather than by the characteristics of its environment.
\end{abstract}

Subject headings: galaxies:bars - galaxies:clusters

\title{
1. INTRODUCTION
}

As Cruzen et al. (2002) have recently emphasized "understanding the role that environment plays in the process of galaxy formation and evolution is one of the most important problems in astrophysics". It would be particularly interesting to know if environment plays a role in the dichotomy between (1) barred and unbarred spiral disks, and (2) between pure spirals and ringed spirals.

Why are some spiral galaxies barred, whereas others do not exhibit bar like structures? Data compiled by Sandage \& Tammann (1981,p.91) show that $26 \%$ of all disk galaxies in the Shapley-Ames catalog exhibit bars, whereas $74 \%$ of all disk systems ${ }^{1}$ do not. It is the purpose of the present investigation to see if environmental factors might affect the fraction

\footnotetext{
${ }^{1}$ Galaxies of Hubble types S0-Sa-Sb-Sc-Sd-Sm-Im are regarded as ordinary disk systems, whereas those of types SB0-SBa-SBb-SBc-SBd-SBm-IBm are regarded as barred disk systems.
} 
of all galaxies that exhibit bars. This question is also of interest in connection with the surprising finding (van den Bergh et al. 1996) that the fraction of barred galaxies appears to drop with increasing lookback time. Detailed simulations (van den Bergh et al. 2002) show that this effect can not be entirely explained by the effects of decreasing resolution, increasing noise, and changes in the wavelength pass band at as a function of increasing redshift. The aim of the present investigation is to see if the decrease in the frequency of barred objects with increasing redshift might be affected by environmental factors. In particular one needs to ask whether the frequency of bars in disk galaxies might depend on environment, i.e. is the frequency of bars for galaxies of a given Hubble type the same in clusters as it is for field galaxies.

The present investigation was based on the use of the morphological classification types given in A Revised Shapley Ames Catalog of Bright Galaxies (Sandage \& Tammann (1981). These data represent the "golden standard" of galaxy classification because they are based on a uniform sample of relatively nearly galaxies observed in blue light with the large reflecting telescopes at the Palomar, Mt. Wilson and Las Campanas observatories. These galaxies were classified by expert morphologists on the system defined by Sandage (1961). For the present investigation it is a particular advantage that Sandage \& Tammann classify galaxies either S (normal) or SB (strongly barred). Numerical experiments show (van den Bergh et al. 2002) that the frequency distribution of such strong bars is little affected by image resolution and noise. On the other hand van den Bergh et al. (2002) have shown that the relative frequency of weak bars [de Vaucouleurs (1959) type SAB and van den Bergh (1960abc) type S(B)] may be strongly affected by image resolution and noise. Such weak bars can literally get lost in the noise. The Shapley-Ames catalog classifications by Sandage \& Tammann therefore represent a robust database for the investigation of the possible dependence of bar frequency on galaxy environment. 
To determine if the frequency of bars is internally determined, or whether it depends

on environment, one first has to establish the nature of the environments of a homogeneous sample of well- classified galaxies. The finest sample of this type is provided by $A$ Revised Shapley-Ames Catalog of Bright Galaxies (Sandage \& Tammann 1981). High quality reproductions of these images have been published by Sandage \& Bedke (1994). Other large collections of morphological classifications of galaxies, such as the The Third Reference Catalogue of de Vaucouleurs et al. (1991), are based on much less homogeneous observational material. Furthermore the advantage of a larger database in the RC3 catalog is offset by the fact that the RC3 galaxies are, in the mean, more distant than those in the Shapley-Ames catalog. Classifications in the RC3 are therefore, on average, based on lower resolution images than are those in A Revised Shapley-Ames Catalog of Sandage \& Tammann.

\section{DETERMINATION OF GALAXY ENVIRONMENT}

Assigning galaxies to a particular environment is a non-trivial problem. For instance the Uppsala General Catalogue of Galaxies (Nilson 1973) mentions companions and group membership in an apparently non-systematic fashion. The catalogs by Zwicky and his collaborators (Zwicky et al. 1961-1968) give detailed lists of galaxy clusterings. However, this information is not very useful for a study of Shapley-Ames galaxies because these clusters are typically an order of magnitude more distant than the Shapley- Ames galaxies that Sandage \& Tammann classified. It was therefore decided to try to assign each Shapley-Ames galaxy to a particular environment by using three criteria: (1) inspection of the prints of the Palomar Sky Survey for the region surrounding each galaxy, (2) by luminosity classification of nearby galaxies (see van den Bergh 1960a), and (3) by making use of available radial velocity information. It should be emphasized that assigning any 
individual galaxy to a particular cluster is a hazardous procedure (e.g. Puddu et al. 2001). With great care one might nevertheless hope to achieve environmental assignments that are meaningful in a statistical sense. For members of the Local Group, which are so nearby that they are spread over the entire sky, membership was taken from van den Bergh (2000). For some nearby groups, such as the M81 cluster, group membership was taken from van den Bergh (1960a). Some uncertainty is introduced in the cluster assignment process by the fact that the internal velocity dispersion in some nearby groups is not small compared to the mean cluster redshift. However, redshift is a relatively reliable distance indicator for the more distant galaxies in the Shapley-Ames catalog. Luminosity classifications (van den Bergh 1960abc) turn out to be useful distance estimators over the entire distance range covered by galaxies in the Shapley- Ames catalog.

Because of the relatively low quality of the Palomar Sky Survey zone at $\delta=-30^{\circ}$ the present investigation was restricted to the area of the sky north of $\delta=-27^{\circ}$. Galaxies were divided into three classes on the basis of their environments: (1) F = Field, (2) G = group 2 and (3) $\mathrm{C}=$ cluster. The assignment of galaxies to groups and clusters was a qualitative procedure that involved both luminosity classifications using the criteria of van den Bergh (1960bc), and the distribution of group and cluster galaxies over the sky. Companions of relatively faint distant Shapley-Ames galaxies were expected to be confined to a small fraction of the area of a single Palamar Sky Survey print, whereas the groups and clusters surrounding a bright Shapley-Ames galaxy might be spread over one or more Palomar Sky Survey prints.

A galaxy was regarded as a member of a group if it appeared to contain at least three

\footnotetext{
${ }^{2}$ Members of the Local Group, which are widely dispersed over the sky, were all assigned to the $\mathrm{G}$ class. For a number of other nearby groups and clusters, which spread over a few Palomar prints, the assignment of membership was taken from van den Bergh (1960a).
} 
other (non-dwarf) members. A galaxy had to have at least six (non-dwarf) members to be assigned to the cluster category. Furthermore clusters were assigned to S, S+E and E categories on the basis of their galaxian content, with $\mathrm{S}$ denoting a cluster in which most galaxies were spirals. Clusters with a mixed population were denoted E+S, and clusters that consisted mainly of elliptical galaxies were denoted by E. On this system the Local Group was regarded as a group $(\mathrm{G})$ with a mixed $(\mathrm{E}+\mathrm{S})$ population. A weaknesses of the adopted classification procedure is, of course, that foreground (or background) galaxies may appear projected on a cluster without being physically associated with it. Since the "field" occupies a much larger area on the sky than do all clusters combined, the probability of a foreground galaxy being projected on a rich cluster is relatively small. Such misassignments would add some noise to any correlations between environment, galaxy type and the existence of bars. An other possible source of bias is that faint companions of distant galaxies near the magnitude limit of the Shapley-Ames survey may have been missed on the Sky Survey prints. As a result some of the most distant cluster members might have been assigned to groups, or even to the field.

The data on which the present investigation was based are listed in Table 1 (field), Table 2 (groups) and in Table 3 (clusters). The galaxy classifications given in these tabulation were taken from Sandage \& Tammann (1981), but suppress secondary information, such as the luminosity classifications, degree of flattening of ellipticals, the presence of rings, etc. The data in Tables 1, 2, and 3 contain information on a total of 391 field galaxies, 145 members of groups, and 394 cluster members that are located in the Shapley-Ames (1932) catalog north of $\delta=-27^{\circ}$. 


\section{SIGNIFICANCE OF ENVIRONMENTAL ASSIGNMENTS}

Since the present assignment of galaxies to field, group, and cluster environments is based on qualitative criteria it is important to see if these environments exhibit clear cut statistical differences in their galactic populations. Data on this point are collected in Table 4. This table lists the frequency with which different morphological galaxy types occur in differing environments. These data clearly show that early-type galaxies occur predominantly in groups and clusters, whereas late-type galaxies predominate in the field. The relative frequency of ellipticals is found to be $\sim 3$ times as great in the cluster areas as it is in the field areas. This reflects the well known dependence of galaxy morphology on environmental galaxy density (Hubble 1936, Dressler 1980). A Kolmogorov-Smirnov test shows that the probability that the frequency distribution of cluster galaxies along the sequence S0-Sa-Sb-Sc-Sd-Sm-Im and SB0-SBa-SBb-SBc-SBd-SBm-IBm was drawn from the same parent population as that for field galaxies only $0.3 \%$. This shows beyond reasonable doubt that the adopted procedures for the assignment of galaixes to three different types different of environments has been highly successful in isolating statistically different environments.

Kraan-Korteweg \& Tammann (1979) have assigned galaxies which they suspected of having distances < $10 \mathrm{Mpc}$ to three environmental classes: (1) field, (2) groups and (3) the Virgo cluster. This differs somewhat from our own environmental assignments since some

of the richest "groups" of Sandage \& Tammann were designated as "clusters" in the present investigation. Nevertheless it is of interest to compare the environmental assignments for the 67 objects that are common to these two investigations. Such a comparison is shown in Table 5. Of 10 galaxies designated as field objects in the present investigation, Kraan-Korteweg \& Tammann assign 8 (80\%) to the field. Of 21 galaxies assigned to groups $20(95 \%)$ are also called group members by Kraan-Korteweg and Tammann. Finally of the 
36 galaxies assigned to clusters in the present investigation Kraan-Korteweg \& Tammann assign $14(39 \%)$ to groups and $19(53 \%)$ to the Virgo cluster. These results show that the present environmental assignments are broadly consistent with those of Kraan-Korteweg \& Tammann.

\section{DEPENDENCE OF BAR FREQUENCY ON ENVIRONMENT}

The Revised Shapley-Ames Catalog of Sandage \& Tammann (1981) presents an almost ideal sample for the study of the frequency of bars in different environments. This is so because: (1) The Shapley-Ames galaxies are bright and therefore, on average, relatively nearby. As a result resolution effects on the visibility of bars are minimized. (2) The Shapley-Ames galaxies were classified on a homogeneous sample of blue sensitive plates obtained with the large reflectors at the Palomar, Mt. Wilson and Las Campanas Observatories. (3) These images were classified in a uniform way by two highly competent morphologists. (4) On the morphological system of Sandage (1961), which was employed by Sandage \& Tammann (1981), Galaxies are classified as being either normal (S), or barred (SB). As a result one need not worry about the possible contamination of the sample by objects exhibiting the week bars that de Vaucouleurs (1959) assigns to class SAB. For a detailed discussion of the effects of resolution on the visibility of bars the reader is referred to Abraham \& Merrifield (2000) and to van den Bergh et al. (2002).

From the data in Table 4 it is found that the frequency of bars for galaxies located in field areas is $25 \pm 3 \%$, compared to $19 \pm 4 \%$ for galaxies in groups, and $28 \pm 3 \%$ for galaxies in clusters. These results suggest that the frequency of bar formation does not depend significantly on galaxy environment. A caveat is, however, that the relative frequency of various galaxy types itself depends on galaxy environment. As a result any systematic dependence of the fraction of barred galaxies on Hubble type might mimic a dependence of 
bar frequency on environment. Table 6 suggests that there is, in fact, little or no systematic variation of bar frequency along the Hubble sequence. As a result the systematic changes in the frequency of galaxy type with environment is not expected to strongly affect the dependence of the fraction of barred galaxies on environment. This problem can be avoided entirely by comparing the relative frequencies of barred and normal spirals of a single Hubble stage. For galaxies of types Sc and SBc the data in Table 4 show that the fraction of barred objects ranges from $17 \pm 3 \%$ in the field, through $12 \pm 5 \%$ in groups to $25 \pm 5 \%$ in clusters. [For the entire Shapley-Ames Catalog Sandage \& Tammann find that $22 \pm 2 \%$ of Sc galaxies are barred.] It is tentatively concluded that presently available data do not show a clear-cut dependence of the fraction of barred galaxies on environment.

\section{CONCLUSIONS}

Inspection of the environments of 930 northern Shapley-Ames galaxies on the prints of the Palomar Sky Survey has made it possible to tentatively assign each of these objects to "field", "group" or "cluster" environments. No significant differences are found between the ratios of barred to non-barrred objects in these different environments. These results suggest that the formation of bars in galaxies is mainly determined by internal factors, rather than by environmental effects.

I am indebted to Roberto Abraham for reawakening my interest in the possible dependence of bar strength on galactic environment. It is also a pleasure to thank Russell Redman for his kind help with the formatting of the tabular material used in this investigation. Note that the full tables 1 through 3 will be available on-line. 
Table 1. NORTHERN SHAPLEY-AMES FIELD GALAXIES.

\begin{tabular}{lll}
\hline \hline Name & Type & Population \\
\hline$N 23$ & $\mathrm{Sb}$ & $\ldots$ \\
$N 24$ & $\mathrm{Sc}$ & $\ldots$ \\
$N 95$ & $\mathrm{Sc}$ & $\ldots$ \\
$N 151$ & $\mathrm{SBbc}$ & $\ldots$ \\
$N 157$ & $\mathrm{Sc}$ & $\ldots$ \\
etc & & \\
\hline
\end{tabular}

Table 2. NORTHERN SHAPLEY-AMES GROUP GALAXIES.

\begin{tabular}{lll}
\hline \hline Name & Type & Population \\
\hline \multirow{N}{*}{$N 45$} & Scd & $\mathrm{S}$ \\
$N 128$ & $S 0$ & $\mathrm{E}$ \\
$N 147$ & $\mathrm{dE}$ & $\mathrm{E}+\mathrm{S}$ \\
$N 185$ & $\mathrm{dE}$ & $\mathrm{E}+\mathrm{S}$ \\
$N 205$ & $S 0 / E 5$ & $\mathrm{E}+\mathrm{S}$ \\
etc & & \\
\hline
\end{tabular}


Table 3. NORTHERN SHAPLEY-AMES CLUSTER GALAXIES.

\begin{tabular}{lll}
\hline \hline & & \\
Name & Type & Population \\
\hline N 16 & SB0 & E \\
N 227 & E & E+S \\
N 237 & Sc & E+S \\
N 245 & Sbc & E + S \\
N 357 & SBa & E \\
etc & & \\
\hline
\end{tabular}


Table 4. FREQUENCY OF MORPHOLOGICAL TYPES IN VARIOUS ENVIRONMENTS

\begin{tabular}{|c|c|c|c|}
\hline Type & Field & Groups & Clusters \\
\hline $\mathrm{E}$ & 20 & 21 & 61 \\
\hline $\mathrm{E} / \mathrm{S} 0$ & 2 & 3 & 10 \\
\hline S0 & 24 & 18 & 40.5 \\
\hline $\mathrm{S} 0 / \mathrm{a}$ & 5 & 2 & 7 \\
\hline $\mathrm{Sa}$ & 27.5 & 7.5 & 24 \\
\hline Sab & 13 & 1.5 & 18 \\
\hline $\mathrm{Sb}$ & 46 & 16 & 21.5 \\
\hline $\mathrm{Sbc}$ & 27 & 7 & 21 \\
\hline $\mathrm{Sc}$ & 120 & 36.5 & 72 \\
\hline Scd & 2 & 2 & 6 \\
\hline $\mathrm{Sd}$ & 0 & 1 & 8 \\
\hline $\mathrm{Sm}$ & 3 & 0.5 & 3.5 \\
\hline $\operatorname{Im}$ & 0 & 1 & 1 \\
\hline SB0 & 9 & 1 & 19 \\
\hline $\mathrm{SB} 0 / \mathrm{a}$ & 2 & 1 & 5 \\
\hline $\mathrm{SBa}$ & 9.5 & 4 & 9.5 \\
\hline SBab & 4 & 0.5 & 1 \\
\hline $\mathrm{SBb}$ & 12 & 7 & 14.5 \\
\hline $\mathrm{SBbc}$ & 24 & 1 & 11 \\
\hline $\mathrm{SBc}$ & 23 & 5 & 24.5 \\
\hline
\end{tabular}


Table 4-Continued

\begin{tabular}{lrrr}
\hline \hline Type & Field & Groups & Clusters \\
\hline SBcd & 2 & 0 & 1 \\
SBd & 0 & 1 & 1 \\
SBm & 2 & 1 & 2 \\
Other & 14 & 5.5 & 12 \\
\hline Total & 391 & 145 & 394 \\
\hline
\end{tabular}


Table 5. COMPARISON OF PRESENT ENVIRONMENTAL ASSIGNMENTS TO THOSE OF KRAAN-KORTEWEG \& TAMMANN (1979)

\begin{tabular}{lrrr}
\hline \hline vdB type & F & G & C \\
\hline K-K\&T type & & & \\
Field & 8 & 1 & 3 \\
Group & 2 & 20 & 14 \\
Virgo & 0 & 0 & 19 \\
\hline
\end{tabular}

Table 6. DEPENDENCE OF BAR

FRACTION ON HUBBLE STAGE a

\begin{tabular}{ll}
\hline \hline \multicolumn{1}{c}{ Type } & Barred \\
\hline S0+S0/a & $31 \pm 4 \%$ \\
Sa+Sab & $25 \pm 4 \%$ \\
Sb+Sbc & $34 \pm 3 \%$ \\
Sc & $21 \pm 2 \%$ \\
Scd-Im & $30 \pm 7 \%$ \\
\hline
\end{tabular}

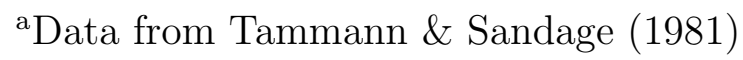




\section{APPENDIX}

\section{RELATIVE FREQUENCY OF FIELD, GROUP, AND CLUSTER GALAXIES}

The statistics in the present paper are affected by the fact that the rich Virgo cluster contributes significantly to the data on the northern Shapley-Ames galaxies. Sandage \& Tammann (1981) find that 108 of the galaxies in the Shapley-Ames Catalog are located within $10^{\circ}$ of the center of the Virgo cluster. Data on the numbers of field, group, and cluster galaxies in the northern Shapley-Ames catalog with, and without, the contribution

from the Virgo cluster are listed in Table 6. This table show that, after excluding the Virgo region, $48 \%$ of all galaxies appear to be members of the field, compared to $18 \%$ located in groups, and $35 \%$ situated in clusters. 
Table 7. FREQUENCY OF FIELD, GROUP, AND CLUSTER MEMBERS

\begin{tabular}{ccccc}
\hline \hline \multirow{2}{*}{ Environment } & \multicolumn{2}{l}{ All galaxies } & \multicolumn{2}{l}{ Virgo region excluded } \\
& No. & $(\%)$ & No. & $(\%)$ \\
\hline \multirow{2}{*}{ Field } & 391 & $(42)$ & 391 & $(48)$ \\
Group & 145 & $(16)$ & 145 & $(18)$ \\
Cluster & 394 & $(42)$ & 286 & $(35)$ \\
\hline
\end{tabular}




\section{REFERENCES}

Abraham, R.G., \& Merrifield, M. R. 2000, AJ, 120, 2842

Cruzen, S., Wehr, T., Weistrop, D., Angione, R. J., \& Hoopes, C. 2002, AJ, 123, 142

de Vaucouleurs, G. 1959, Handbuch de Physik, 53, 275

de Vaucouleurs, G., de Vaucouleurs, A., Corwin, H.G., Buta, R. J., Paturel, G., Fouque, P. 1991, The Third Reference Catalogue of Bright Galaxies, (Berlin: Springer Verlag)

Dressler, A. 1980, ApJ, 236, 351

Hubble, E. 1936, The Realm of the Nebulae, (New Haven: Yale Univ. Press), p. 79

Kraan-Korteweg, R. C., \& Tammann, G. A., 1979, Astr. Nachr. 300, 181

Nilson, P. 1973, Uppsala General Cataglogue of Galaxies, (Uppsala: Uppsala Offset Center)

Puddu, E., Andreon, S. Longo, G., Strazzullo, V., Paolillo, M., \& Gal, R. R. 2001, A\&A, 379,426

Sandage, A. 1961, The Hubble Atlas of Galaxies, (Washington: The Carnegie Institution of Washington)

Sandage, A. \& Bedke, J. 1994, The Carnegie Atlas of Galaxies, (Washington: Carnegie Institution of Washington)

Sandage, A., \& Tammann, G.A. 1981, A Revised Shapley-Ames Catalog of Bright Galaxies,(Washington: Carnegie Institution of Washington)

Shapley, H., \& Ames, A. 1932, Harvard Ann. Vol. 88, No. 2

van den Bergh, S. 1960a, Publ. David Dunlap Obs. Vol. 2, No. 6, p.159

van den Bergh, S. 1960b, ApJ, 131, 215

van den Bergh, S. 1960c, ApJ, 131, 558 
van den Bergh, S. 2000, The Galaxies of the Local Group, (Cambridge: Cambridge University Press)

van den Bergh, S., Abraham, R. G., Ellis, R. S., Tanvir, E. R., Santiago, B. X., \& Glazebrook, K. G. 1996, AJ, 112, 359

van den Bergh, S., Abraham, R. G., Whyte, L. F., Merrifield, M. R., Eskridge, P., Frogel, J. A., \& Pogge, R. 2002, AJ, 123, xxxx 